\title{
Synthesis and Antitumor Activity of the Thiazoline and Thiazine Multithioether
}

\author{
Wei Wang ${ }^{12^{*}}$, Bing $\mathrm{Zhao}^{3}$, Chao $\mathrm{Xu}^{2}$, Wenpeng $\mathrm{Wu}^{2}$ \\ ${ }^{1}$ School of Perfume and Aroma Technology, Shanghai Institute of Technology, Shanghai, China \\ ${ }^{2}$ School of Chemical Engineering, University of Science and Technology Liaoning, Anshan, China \\ ${ }^{3}$ Institute of Chemical and Chemistry Engineering, Qiqihar University, Qiqihar, China \\ Email: *wangweittg@163.com
}

Received March 30, 2012; revised April 14, 2012; accepted May 9, 2012

\begin{abstract}
A series of novel multithioether derivatives were synthesized by the combination of thiazoline and thiazine with dibromides and their structures were characterized by IR, ${ }^{1} \mathrm{H}$ NMR, MS and elemental analysis. The synthesized derivatives were tested for antitumor activity.
\end{abstract}

Keywords: Thiazoline; Thiazine; Multithioether; Synthesis; Antitumor Activity

\section{Introduction}

Nitrogen-containing five- or six-membered heterocyclic compounds such as oxazolines, thiazolines and thiazines are of great interest to organic chemists, because they are present in various natural compounds having interesting bioactivities [1-3]. Furthermore, the optically active heterocyclic compounds have been successfully used in asymmetric synthesis as chiral templates [4,5] or ligands [6-9] reported the synthesis of thioethers and thioesters in acetonitrile. Herein, we reported an efficient synthesis of some thiazoline and thiazine multithioethers in a friendly solvent-ethanol and explored the biological activities of the obtained target products against A549 (human lung cancer cell) and Bcap-37 (human breast cancer cell).

\section{Results and Discussion}

The raw materials thiazolidine-2-thione (1) and 1,3-thiazinane-2-thione (2) were synthesized according to the literature method $[10,11]$. Then they were treated with different dibromides under different reaction conditions. In the process of experiments, different reaction factors were examined to obtain the optimum reaction conditions such as the reaction temperature, the reaction time, employed bases and solvent and mixing sequence. The results showed that the start materials thiazolidine-2-thione (1) and 1,3-thiazinane-2-thione (2) should be activated by solid $\mathrm{KOH}$ and dissolved firstly in order to ensure that the subsequent reaction was fully carried out. The general reaction procedure was as follows (Scheme 1). To an

"Corresponding author. anhydrous ethanol solution $(3 \mathrm{~mL})$ of the heterocycle 2-thione (4.4 mmol) (1 or 2), $\mathrm{KOH}(0.25 \mathrm{~g}, 4.4 \mathrm{mmol})$ was added. The suspension was stirred until the solution was clarified. Then, the ethanol solution $(2 \mathrm{~mL})$ of different dibromides (3) $(2.1 \mathrm{mmol})$ was slowly added and a white precipitate was obtained gradually. Then the precipitates were filtered, washed with ethanol, and then dried after. The crude products were recrystallized from water and white products were obtained.

The target compounds were characterized by the spectra. Spectroscopic data were in agreement with the desired structures. For example, in terms of the ${ }^{1} \mathrm{H}$ NMR spectra of compound $\mathbf{4}$, the two protons of the methylene attached to $\mathrm{S}$ atom appeared as a singlet at 4.34 and 4.47 $\mathrm{ppm}$ in compounds $\mathbf{4 e}$ and $\mathbf{4 f}$, respectively; which the two protons of the methylene were splitted as a triplet and a doublet at 3.26 and $3.74 \mathrm{ppm}$ in compounds $\mathbf{4 c}$ and 4d respectively. The data of IR, MS and element analysis further confirmed their structures of the target products.

\section{Biological Tests}

The in vitro antitumor activities of the synthesized target compounds against A-549 (human lung cancer cell) and Bcap-37 (human breast cancer cell) were evaluated by the standard MTT assay (Table 1) [12]. The data revealed that compounds $\mathbf{4 g}$ and $\mathbf{5 g}$ possessed higher antitumor activities. $\mathrm{IC}_{50}$ values against A-549 and Bcap-37 of compound $4 \mathrm{~g}$ were 22.58 and $19.41 \mu \mathrm{g} / \mathrm{mL}$, corresponding the values of compound $\mathbf{5 g}$ were 8.26 and 9.30 $\mu \mathrm{g} / \mathrm{mL}$, respectively. As compared with compounds $\mathbf{4 a}$ $\mathbf{4 g}$, compounds $5 \mathrm{a}-\mathbf{5 g}$ exhibit higher antitumor activities. 
<smiles>FCCCSC1NCCS1</smiles><smiles>[R]Br</smiles>
KOH, Ethanol r.t. 3a-g .<smiles>[R]SC1=NCCS1</smiles><smiles>[R]SC1=NCCCS1</smiles>

5a-g<smiles>C#CCCCCCC</smiles><smiles>C=CCc1ccc(CC)cc1</smiles><smiles>CCc1ccccc1CC</smiles><smiles>CCc1cccc(CC)c1</smiles>

Scheme 1. Synthesis of the thiazoline and thiazine multithioether.

Table 1. $\mathrm{IC}_{50}$ data of A-549 and Bcap-37 of in vitro for the target compounds $4 \mathrm{a}-4 \mathrm{~g}$ and $5 \mathrm{a}-5 \mathrm{~g}$.

\begin{tabular}{|c|c|c|c|c|c|c|c|}
\hline \multirow{2}{*}{ Compd. } & \multirow{2}{*}{ F.W. } & \multicolumn{2}{|c|}{$\mathrm{IC}_{50}(\mu \mathrm{g} / \mathrm{mL})$} & \multirow{2}{*}{ Compd. } & \multirow{2}{*}{ F.W. } & \multicolumn{2}{|c|}{$\mathrm{IC}_{50}(\mu \mathrm{g} / \mathrm{mL})$} \\
\hline & & A-549 & Bcap-37 & & & A-549 & Bcap-37 \\
\hline $4 a$ & 264 & 105.02 & 105.23 & $5 \mathbf{a}$ & 292 & 90.05 & 89.30 \\
\hline $4 b$ & 278 & 74.36 & 80.30 & $5 \mathbf{b}$ & 306 & 71.45 & 55.29 \\
\hline $4 c$ & 292 & 50.68 & 30.46 & $5 c$ & 320 & 35.34 & 20.03 \\
\hline $4 d$ & 290 & 45.28 & 41.79 & $5 d$ & 318 & 20.57 & 20.17 \\
\hline $4 e$ & 340 & 36.17 & 40.25 & $5 e$ & 368 & 22.86 & 10.56 \\
\hline $4 f$ & 340 & 35.57 & 30.88 & $5 f$ & 368 & 10.13 & 15.30 \\
\hline $4 g$ & 340 & 22.58 & 19.41 & $5 g$ & 368 & 8.26 & 9.30 \\
\hline
\end{tabular}

\section{Experimental}

All chemicals and solvents were purchased from Sinopharm, Beijing Chemical Plant. Melting points were recorded on a Digital Melting Point Apparatus. Elemental analysis of carbon, hydrogen and nitrogen were determined on a Flash-1112 series elemental analyser. IR spectra $(\mathrm{KBr})$ were recorded on a Perkin Elmer FTIR. The ${ }^{1} \mathrm{H}$ NMR and spectra were measured at $25^{\circ} \mathrm{C}$ on a Bruker Avance-500 NMR spectrometer respectively in DMSO- $\mathrm{d}_{6}$ solution using TMS as an internal reference. MS spectra were recorded on an Agilent $1100 \mathrm{LC} / \mathrm{MS}$.

Synthesis of thiazoline multithioethers $4 \mathrm{a}-4 \mathrm{~g}$ and 5a - 5g:

Synthesis of 1,2-Bis(2-thiazolin-2-ylsulfanyl) ethane (4a). To an anhydrous ethanol solution $(3 \mathrm{~mL})$ of the heterocyclic 2-thione (4.4 mmol), $\mathrm{KOH}(0.25 \mathrm{~g}, 4.4 \mathrm{mmol})$ was added. The suspension was stirred until the solution was clarified. Then, the ethanol solution $(2 \mathrm{~mL})$ of different dibromides $(3 \mathrm{a}-3 \mathrm{~g})(2.1 \mathrm{mmol})$ was slowly added and a white precipitate was obtained gradually, continue to react to $72 \mathrm{~h}$. Then the precipitates were filtered, washed with ethanol, and then dried. The crude products were recrystallized from water and white products were obtained (4a). Yield (0.37 g, 69.5\%); M.p. $125^{\circ} \mathrm{C}-127^{\circ} \mathrm{C}$.

The other compounds of this series $4 \mathrm{~b}-4 \mathrm{~g}$ and $5 \mathrm{a}-5 \mathrm{~g}$ were prepared adopting the above procedure.

The physical and spectral data of the compounds $4 \mathrm{a}-$ $4 \mathrm{~g}$ and $5 \mathrm{a}-5 \mathrm{~g}$.

1,2-Bis(2-thiazolin-2-ylsulfanyl)ethane(4a):

IR spectrum, $v, \mathrm{~cm}^{-1}: 2984,2952,2848(\mathrm{CH}), 1567$ $(\mathrm{C}=\mathrm{N}), 1450\left(\gamma_{\mathrm{CH}_{2}}\right) .{ }^{1} \mathrm{H}$ NMR spectrum $(500 \mathrm{MHz}$, DMSO$\left.\mathrm{d}_{6}\right), \delta, \operatorname{ppm}(\mathrm{J}, \mathrm{Hz}): 4.22\left(4 \mathrm{H}, \mathrm{t}, \mathrm{CH}_{2}\right), 3.44\left(4 \mathrm{H}, \mathrm{t}, \mathrm{CH}_{2}\right)$, $3.41\left(4 \mathrm{H}, \mathrm{t}, \mathrm{CH}_{2}\right)$. MS, m/z: $263\left(\mathrm{M}^{+}\right), 265(\mathrm{M}+2)$. Found, \%: C 36.64; H 4.45; N 10.66; S 48.70. Calculated, 
\%: C 36.50; H 4.56; N 10.65; S 48.67.

\section{1,3-Bis(2-thiazolin-2-ylsulfanyl)propane(4b):}

Yield, $56.2 \% ; \mathrm{mp} 211^{\circ} \mathrm{C}-212^{\circ} \mathrm{C}$; IR spectrum, $v, \mathrm{~cm}^{-1}$ : 2928, 2840 $(\mathrm{CH}), 1566(\mathrm{C}=\mathrm{N}), 1432\left(\gamma_{\mathrm{CH}_{2}}\right) ;{ }^{1} \mathrm{H}$ NMR spectrum $\left(500 \mathrm{MHz}, \mathrm{DMSO}-\mathrm{d}_{6}\right), \delta, \operatorname{ppm}(\mathrm{J}, \mathrm{Hz}): 2.82$ $(8 \mathrm{H}, \mathrm{m}, \mathrm{CH}), 2.67\left(2 \mathrm{H}, \mathrm{m}, \mathrm{CH}_{2}\right), 1.98\left(4 \mathrm{H}, \mathrm{t}, \mathrm{CH}_{2}\right)$; MS, m/z: $277\left(\mathrm{M}^{+}\right), 279(\mathrm{M}+2)$. Found, \%: C 38.96; H 5.07; N 10.07; S 46.30. Calculated, \%: C 38.99; H 5.05; N 10.11; S 46.21.

\section{1,4-Bis(2-thiazolin-2-ylsulfanyl)butane(4c):}

Yield, $71.8 \%$; $\mathrm{mp} 108^{\circ} \mathrm{C}-110^{\circ} \mathrm{C}$; IR spectrum, $v, \mathrm{~cm}^{-1}$ : 2920, 2830 $(\mathrm{CH}), 1556(\mathrm{C}=\mathrm{N}), 1432\left(\gamma_{\mathrm{CH}_{2}}\right) .{ }^{1} \mathrm{H}$ NMR spectrum $\left(500 \mathrm{MHz}, \mathrm{DMSO}-\mathrm{d}_{6}\right), \delta, \operatorname{ppm}(J, \mathrm{~Hz}): 4.23$ $\left(4 \mathrm{H}, \mathrm{t}, \mathrm{CH}_{2}\right), 3.41\left(4 \mathrm{H}, \mathrm{t}, \mathrm{CH}_{2}\right), 3.26\left(4 \mathrm{H}, \mathrm{t}, \mathrm{CH}_{2}\right), 1.93$ $\left(4 \mathrm{H}, \mathrm{m}, \mathrm{CH}_{2}\right)$; MS, m/z: $291\left(\mathrm{M}^{+}\right), 293(\mathrm{M}+2)$. Found, \%: C 41.31; H 5.60; N 9.58; S 43.97. Calculated, \%: C 41.24; H 5.51; N 9.62; S 43.97.

trans-1,4-Bis(2-thiazolin-2-ylsulfanyl)butene(4d):

Yield, $83.9 \% ; \mathrm{mp} 48^{\circ} \mathrm{C}-52^{\circ} \mathrm{C}$; IR spectrum, $v \mathrm{~cm}^{-1}$ : 2924, 2844(CH), 1560 $(\mathrm{C}=\mathrm{N}), 1402\left(\gamma_{\mathrm{CH}_{2}}\right) .{ }^{1} \mathrm{H} \mathrm{NMR}$ spectrum $\left(500 \mathrm{MHz}, \mathrm{DMSO}-\mathrm{d}_{6}\right), \delta, \operatorname{ppm}(\mathrm{J}, \mathrm{Hz}): 5.81$ $\left(2 \mathrm{H}, \mathrm{t}, \mathrm{CH}_{2}\right), 4.22\left(4 \mathrm{H}, \mathrm{t}, \mathrm{CH}_{2}\right), 3.74\left(4 \mathrm{H}, \mathrm{d}, \mathrm{CH}_{2}\right), 3.40$ $\left(4 \mathrm{H}, \mathrm{t}, \mathrm{CH}_{2}\right) . \mathrm{MS}, \mathrm{m} / \mathrm{z}$ : $289\left(\mathrm{M}^{+}\right), 291(\mathrm{M}+2)$. Found, \%: C 41.50; H 4.87; N 9.70; S 44.25. Calculated, \%: C 41.52; H 4.84; N 9.69; S 44.29.

\section{1,4-Bis(2-thiazolin-2-ylsulfanyl)toluene(4e):}

Yield, $61.8 \%$; $\mathrm{mp} 62^{\circ} \mathrm{C}-64^{\circ} \mathrm{C}$; IR spectrum, $v \mathrm{~cm}^{-1}$ : 3062, 2932, 2844(CH), 1592(C=N), 1422 $\left(\gamma_{\mathrm{CH}_{2}}\right)$. ${ }^{1} \mathrm{H}$ NMR spectrum $\left(500 \mathrm{MHz}, \mathrm{DMSO}-\mathrm{d}_{6}\right), \delta, \operatorname{ppm}(J, \mathrm{~Hz}): 7.28$ (4H, m, ArH), 4.34 (4H, s, $\left.\mathrm{CH}_{2}\right), 4.23\left(4 \mathrm{H}, \mathrm{t}, \mathrm{CH}_{2}\right), 3.41$ $\left(4 \mathrm{H}, \mathrm{t}, \mathrm{CH}_{2}\right) . \mathrm{MS}, \mathrm{m} / \mathrm{z}: 339\left(\mathrm{M}^{+}\right), 341(\mathrm{M}+2)$. Found, \%: C 49.55; H 4.75; N 8.22; S 37.79. Calculated, \%: C 49.56; H 4.72; N 8.26; S 37.76 .

\section{1,2-Bis(2-thiazolin-2-ylsulfanyl)toluene(4f):}

Yield, $58 \%$; mp $112^{\circ} \mathrm{C}-114^{\circ} \mathrm{C}$; IR spectrum, $v \mathrm{~cm}^{-1}$ : 3062, 2982, 2846(CH), 1556(C=N), 760. ${ }^{1} \mathrm{H}$ NMR spectrum $\left(500 \mathrm{MHz}, \mathrm{DMSO}-\mathrm{d}_{6}\right), \delta, \operatorname{ppm}(\mathrm{J}, \mathrm{Hz}): 7.36(2 \mathrm{H}, \mathrm{m}$, ArH), 7.21 (2H, m, ArH), $4.47\left(4 \mathrm{H}, \mathrm{s}, \mathrm{CH}_{2}\right), 4.22(4 \mathrm{H}, \mathrm{t}$, $\left.\mathrm{CH}_{2}\right), 3.39\left(4 \mathrm{H}, \mathrm{t}, \mathrm{CH}_{2}\right)$. MS, $\mathrm{m} / \mathrm{z}: 340\left(\mathrm{M}^{+}\right), 342(\mathrm{M}+2)$. Found, \%: C 49.40; H 4.72; N 8.25; S 37.62. Calculated, $\%$ : C 49.41; H 4.71; N 8.23; S 37.65.

\section{1,3-Bis(2-thiazolin-2-ylsulfanyl)toluene(4g):}

Yield, 61.7\%; mp $62^{\circ} \mathrm{C}-63^{\circ} \mathrm{C}$; IR spectrum, $v, \mathrm{~cm}^{-1}$ : 3061, 2982, 2846(CH), 1556(C=N), 790. ${ }^{1} \mathrm{H}$ NMR spectrum $\left(500 \mathrm{MHz}, \mathrm{DMSO}-\mathrm{d}_{6}\right), \delta, \operatorname{ppm}(\mathrm{J}, \mathrm{Hz}): 7.26(4 \mathrm{H}, \mathrm{m}$, $\mathrm{ArH}), 4.34\left(4 \mathrm{H}, \mathrm{s}, \mathrm{CH}_{2}\right), 4.23\left(4 \mathrm{H}, \mathrm{t}, \mathrm{CH}_{2}\right), 3.41(4 \mathrm{H}, \mathrm{t}$, $\left.\mathrm{CH}_{2}\right)$. MS, m/z: $339\left(\mathrm{M}^{+}\right), 341(\mathrm{M}+2)$. Found, \%: C 49.53; H 4.70; N 8.22; S 37.79. Calculated, \%: C 49.55; H 4.72; N 8.27; S 37.78.

\section{1,2-Bis(4,5-Dihydro-1,3-thiazin-2-ylsulfanyl)ethane(} 5a):

Yield, $71.3 \%$; mp $72^{\circ} \mathrm{C}-73^{\circ} \mathrm{C}$; IR spectrum, $v, \mathrm{~cm}^{-1}$ : 2940, 2835(CH), 1602(C=N), 1452( $\left.\gamma_{\mathrm{CH}_{2}}\right)$. ${ }^{1} \mathrm{H}$ NMR spectrum $\left(500 \mathrm{MHz}, \mathrm{DMSO}-\mathrm{d}_{6}\right), \delta, \operatorname{ppm}(J, \mathrm{~Hz}): 3.76$
(4H, t, $\left.\mathrm{CH}_{2}\right), 3.19\left(4 \mathrm{H}, \mathrm{t}, \mathrm{CH}_{2}\right), 3.08\left(4 \mathrm{H}, \mathrm{t}, \mathrm{CH}_{2}\right), 1.92$ $\left(4 \mathrm{H}, \mathrm{t}, \mathrm{CH}_{2}\right), 1.89\left(4 \mathrm{H}, \mathrm{m}, \mathrm{CH}_{2}\right) ; \mathrm{MS}, \mathrm{m} / \mathrm{z}: 291\left(\mathrm{M}^{+}\right), 293$ $(\mathrm{M}+2)$. Found, \%: C 41.21; H 5.51; N 9.59; S 43.95. Calculated, \%: C 41.23; H 5.50; N9.64; S 43.96.

1,3-Bis(4,5-Dihydro-1,3-thiazin-2-ylsulfanyl)propane (5b):

Yield, $27.02 \% ; \mathrm{mp} 267^{\circ} \mathrm{C}-268^{\circ} \mathrm{C}$; IR spectrum, $v$, $\mathrm{cm}^{-1}: 2925,2850(\mathrm{CH}), 1590(\mathrm{C}=\mathrm{N}), 1425\left(\gamma_{\mathrm{CH}_{2}}\right) .{ }^{1} \mathrm{H} \mathrm{NMR}$ spectrum $\left(500 \mathrm{MHz}, \mathrm{DMSO}-\mathrm{d}_{6}\right), \delta$, ppm $(J, \mathrm{~Hz}): 2.92(8 \mathrm{H}$, $\left.\mathrm{m}, \mathrm{CH}_{2}\right), 2.67\left(2 \mathrm{H}, \mathrm{m}, \mathrm{CH}_{2}\right), 2.08\left(4 \mathrm{H}, \mathrm{t}, \mathrm{CH}_{2}\right) . \mathrm{MS}, \mathrm{m} / \mathrm{z}$ : $305\left(\mathrm{M}^{+}\right), 307(\mathrm{M}+2)$. Found, \%: C 43.29; H 5.88; N 9.22; S 42.01. Calculated, \%: C 43.28; H 5.90; N 9.18; S 41.97 .

1,4-Bis(4,5-Dihydro-1,3-thiazin-2-ylsulfanyl)butane (5c): Yield, 50.2\%; mp $72^{\circ} \mathrm{C}-73^{\circ} \mathrm{C}$; IR spectrum, $v, \mathrm{~cm}^{-1}$ : 2940, 2846(CH), 1602(C=N), 1452( $\left.\gamma_{\mathrm{CH}_{3}}\right) .{ }^{1} \mathrm{H}$ NMR spectrum $\left(500 \mathrm{MHz}, \mathrm{DMSO}-\mathrm{d}_{6}\right), \delta$, ppm $(J, \mathrm{~Hz}): 3.76(4 \mathrm{H}$, t, $\left.\mathrm{CH}_{2}\right), 3.19\left(4 \mathrm{H}, \mathrm{t}, \mathrm{CH}_{2}\right), 3.08\left(4 \mathrm{H}, \mathrm{t}, \mathrm{CH}_{2}\right), 1.92(4 \mathrm{H}, \mathrm{t}$, $\left.\mathrm{CH}_{2}\right), 1.89\left(4 \mathrm{H}, \mathrm{m}, \mathrm{CH}_{2}\right)$. MS, m/z: $319\left(\mathrm{M}^{+}\right), 321(\mathrm{M}+2)$. Found, \%: C 45.12; H 6.29; N 8.76; S 40.11. Calculated, $\%$ : C 45.14; H 6.27; N 8.78; S 40.13 .

\section{1,4-Bis(4,5-Dihydro-1,3-thiazin-2-ylsulfanyl)butene} (5d):

Yield, $57.6 \%$; $\mathrm{mp} 84^{\circ} \mathrm{C}-86^{\circ} \mathrm{C}$; IR spectrum, $v, \mathrm{~cm}^{-1}$ : 2914, 2842(CH), $1600(\mathrm{C}=\mathrm{N}), 1424\left(\gamma_{\mathrm{CH}_{2}}\right)$. ${ }^{1} \mathrm{H}$ NMR spectrum $\left(500 \mathrm{MHz}, \mathrm{DMSO}-\mathrm{d}_{6}\right), \delta, \operatorname{ppm}(J, \mathrm{~Hz}): 5.68(2 \mathrm{H}$, $\mathrm{m}, \mathrm{CH}=\mathrm{CH}), 3.75\left(4 \mathrm{H}, \mathrm{t}, \mathrm{CH}_{2}\right), 3.61\left(4 \mathrm{H}, \mathrm{d}, \mathrm{CH}_{2}\right)$, 3.06(4H, t, $\left.\mathrm{CH}_{2}\right), 1.92\left(2 \mathrm{H}, \mathrm{m}, \mathrm{CH}_{2}\right)$. MS, $\mathrm{m} / \mathrm{z}: 317\left(\mathrm{M}^{+}\right)$, 319 (M + 2). Found, \%: C 45.44; H 5.64; N 8.85; S 40.39. Calculated, \%: C 45.43; H5.68; N 8.83; S 40.38.

\section{1,4-Bis(4,5-Dihydro-1,3-thiazin-2-ylsulfanyl)toluene} (5e):

Yield, $65.7 \%$; mp $76^{\circ} \mathrm{C}-77^{\circ} \mathrm{C}$; IR spectrum, $v, \mathrm{~cm}^{-1}$ : 3060, 2922, 2838(CH), 1591 $(\mathrm{C}=\mathrm{N}), 862 .{ }^{1} \mathrm{H}$ NMR spectrum $\left(500 \mathrm{MHz}, \mathrm{DMSO}-\mathrm{d}_{6}\right), \delta$, ppm $(\mathrm{J}, \mathrm{Hz}): 7.25(4 \mathrm{H}, \mathrm{m}$, ArH), $4.21\left(4 \mathrm{H}, \mathrm{s}, \mathrm{CH}_{2}\right), 3.77\left(4 \mathrm{H}, \mathrm{t}, \mathrm{CH}_{2}\right), 3.06(4 \mathrm{H}, \mathrm{t}$, $\left.\mathrm{CH}_{2}\right), 1.92\left(4 \mathrm{H}, \mathrm{t}, \mathrm{CH}_{2}\right) . \mathrm{MS}, \mathrm{m} / \mathrm{z}: 367\left(\mathrm{M}^{+}\right), 369(\mathrm{M}+2)$; Found, \%: C 52.35; H 5.40; N 7.67; S 34.85. Calculated, \%: C 52.32; H 5.45; N 7.63; S 34.88.

\section{1,2-Bis(4,5-Dihydro-1,3-thiazin-2-ylsulfanyl)toluene (5f):}

Yield, $65.2 \%$; $\mathrm{mp} 40^{\circ} \mathrm{C}-41^{\circ} \mathrm{C}$; IR spectrum, $v, \mathrm{~cm}^{-1}$ : 3062, 2920, 2854(CH), 1587(C=N), 750. ${ }^{1} \mathrm{H}$ NMR spectrum $\left(500 \mathrm{MHz}, \mathrm{DMSO}-\mathrm{d}_{6}\right), \delta, \operatorname{ppm}(\mathrm{J}, \mathrm{Hz}): 7.24(4 \mathrm{H}, \mathrm{m}$, $\mathrm{ArH}), 4.16\left(4 \mathrm{H}, \mathrm{s}, \mathrm{CH}_{2}\right), 3.78\left(4 \mathrm{H}, \mathrm{t}, \mathrm{CH}_{2}\right), 3.20(4 \mathrm{H}, \mathrm{t}$, $\left.\mathrm{CH}_{2}\right), 1.85\left(4 \mathrm{H}, \mathrm{m}, \mathrm{CH}_{2}\right) . \mathrm{MS}, \mathrm{m} / \mathrm{z}: 367\left(\mathrm{M}^{+}\right), 369(\mathrm{M}+$ 2). Found, \%: C 52.34; H 5.44; N 7.65; S 34.83. Calculated, \%: C, 52.33; H, 5.42; N, 7.65; S, 34.85.

\section{1,3-Bis(4,5-Dihydro-1,3-thiazin-2-ylsulfanyl)toluene} (5g):

Yield, $62.3 \%$; $\mathrm{mp} 43^{\circ} \mathrm{C}-44^{\circ} \mathrm{C}$; IR spectrum, $v, \mathrm{~cm}^{-1}$ : 3062, 2918, 2842(CH), 1594(C=N), 703. ${ }^{1} \mathrm{H}$ NMR spectrum $\left(500 \mathrm{MHz}, \mathrm{DMSO}-\mathrm{d}_{6}\right), \delta$, ppm $(\mathrm{J}, \mathrm{Hz}): 7.21(4 \mathrm{H}, \mathrm{m}$, ArH), $4.20\left(4 \mathrm{H}, \mathrm{s}, \mathrm{CH}_{2}-\mathrm{H}\right), 3.75\left(4 \mathrm{H}, \mathrm{t}, \mathrm{CH}_{2}\right), 3.11(4 \mathrm{H}, \mathrm{t}$, 
$\left.\mathrm{CH}_{2}\right), 1.90\left(4 \mathrm{H}, \mathrm{m}, \mathrm{CH}_{2}\right) . \mathrm{MS}, \mathrm{m} / \mathrm{z}: 367\left(\mathrm{M}^{+}\right), 369(\mathrm{M}+$ 2). Found, \%: C 52.34; H 5.42; N 7.68; S 34.86. Calculated, \%: C 52.34; H 5.43; N 7.65; S 34.88.

\section{Acknowledgements}

The project was supported by the Key Laboratory Project (2008S127) and the Initial Fund for Young Teachers of Qiqihar University (000203).

\section{REFERENCES}

[1] A. Adams and N. De Kimpe, "Chemistry of 2-Acetyl-1pyrroline, 6-Acetyl-1,2,3,4-tetrahydropyridine, 2-Acetyl-2thiazoline, and 5-Acetyl-2,3-dihydro-4H-thiazine: Extraordinary Maillard Flavor Compounds," Chemical Reviews, Vol. 106, No. 6, 2006, pp. 2299-2319. doi:10.1021/cr040097y

[2] D. M. Du, S. F. Lu, T. Fang and J. X. Xu, "Asymmetric Henry Reaction Catalyzed by $C_{2}$-Symmetric Tridentate Bis(oxazoline) and Bis(thiazoline) Complexes: Metal-Controlled Reversal of Enantioselectivity," Journal of Organic Chemistry, Vol. 70, No. 9, 2005, pp. 3712-3715. doi:10.1021/jo050097d

[3] Z. Jin, "Muscarine, Imidazole, Oxazole, and Thiazole Alkaloids," Natural Product Reports, Vol. 20, No. 6, 2003, pp. 584-605. doi:10.1039/b304142p

[4] J. R. Lewis, "Amaryllidaceae, Sceletium, Imidazole, Oxazole, Thiazole, Peptide and Miscellaneous Alkaloids," Natural Product Reports, Vol. 19, No. 2, 2002, pp. 223258. doi:10.1039/b007741k

[5] S. F. Lu, D. M. Du, J. X. Xu and S. W. Zhang, "Asymmetric Michael Addition of Nitroalkanes to Nitroalkenes Catalyzed by $C_{2}$-Symmetric Tridentate Bis(oxazoline) and Bis(thiazoline) Zinc Complexes," Journal of the American Chemical Society, Vol. 128, No. 23, 2006, pp. 7418-
7419. doi: $10.1021 / \mathrm{ja} 0604008$

[6] M. Melchior, K. H. Thompson, J. M. Jong, et al., "Vanadium Complexes as Insulin Mimetic Agents: Coordination Chemistry and in Vivo Studies of Oxovanadium(IV) and Dioxovanadate(V) Complexes Formed from Naturally Occurring Chelating Oxazolinate, Thiazolinate, or Picolinate Units," Inorganic Chemistry, Vol. 38, No. 10, 1999, pp. 2288-2293. doi:10.1021/ic981231y

[7] S. T. A. Shah, K. M. Khan, A. M. Heinrich, et al., "An Alternative Approach towards the Syntheses of Thioethers and Thioesters Using CsF-Celite in Acetonitrile," Tetrahedron Letters, Vol. 43, No. 46, 2002, pp. 8281-8283. doi:10.1016/S0040-4039(02)02028-2

[8] E. Shuter, H. R. Hoveyda, V. Karunaratne, et al., "Bis (ligand) Rhenium(V) and Technetium(V) Complexes of Two Naturally Occurring Binding Moieties (Oxazoline and Thiazoline)," Inorganic Chemistry, Vol. 35, No. 2, 1996, pp. 368-372. doi:10.1021/ic9507528

[9] B. Zhao, Y. Q. Feng and S. S. Zhang, "Novel Synthesis and Characterization of 1,3-Bis(2-dihydrothiazolyl) thiaalkoxy-p-tert-butylcalix[4]arenes," Synthetic Communications, Vol. 37, No. 20, 2007, pp. 3479-3484. doi: $10.1080 / 00397910701555212$

[10] M. Jain, P. Khanna and A. Saxena, "Synthesis of Some Novel Bis-spiro[indole-pyrazolinyl-thiazolidine]-2,4'diones," Synthetic Communications, Vol. 36, 2006, pp. 1863-1872. doi:10.1080/00397910600602560

[11] A. Dandia, M. Saha and B. Rani, "Microwave-Induced Synthesis of Spiro[indoline-3,2'-[1,3]thiazinane]-2,4'diones," Journal of Chemical Research, Synopses, Vol. 7, 1998, pp. 360-361. doi:10.1039/a706678c

[12] X. Zhai, J. Li, L. He, et al., "Synthesis and in Vitro Cytotoxicity of Novel 1,4-Disubstituted Phthalazines," Chinese Chemical Letters, Vol. 19, No. 1, 2008, pp. 29-32. doi:10.1016/j.cclet.2007.11.018 